Fermilab-TM-2312-AD

NuMI-B-926

Version 2.1

July 12,2004

\title{
Studies of Beam Heating of Proton Beam Profile Monitor SEM's
}

\author{
Zarko Pavlovich, Thomas H. Osiecki, Sacha E. Kopp \\ University of Texas at Austin
}

\begin{abstract}
We present calculations of the expected temperature rise of proton beam profile monitors due to beam heating. The profile monitors are secondary emission monitors (SEM's) to be made of Titanium foils. The heating is studied to understand whether there is any loss of tension or alignment of such devices. Additionally, calculations of thermally-induced dynamic stress are presented. Ti foil is compared to other materials and also to wire SEM's. The calculations were initially performed for the NuMI beam, where the per-pulse intensity is quite high; for completeness the calculations are also performed for other beam energies and intensities.
\end{abstract}

\section{Introduction}

Secondary emission monitors (SEM's) will be located in the vacuum of the accelerator beamline and are to be used to measure the NuMI primary beam profile and halo. A total of 10 monitors are to be located along the primary beam line. The monitors must be sensitive to beam spots of order $1 \mathrm{~mm}$ and be accurate in beam centroid measurements to within $50 \mu \mathrm{m}$.

Secondary electron emission is a surface phenomenon in which $20-100 \mathrm{eV}$ electrons are ejected from a metal through which passes a charged particle [1]. Such emission occurs with great frequency for highly charged, slow moving charged particle beams, but decreases to approximately $1-5 \%$ of an electron per multi-GeV proton[2]. SEM devices may be used for extracted beam lines, however, because of the large $4 \times 10^{13}$ protons/pulse expected will result in a large detectable ejected charge from the SEM.

The NuMI profile monitors consist of $X$ and $Y$ foil planes, each of which is segmented into strips. In this way, the relative secondary electron emission from each strip or wire within a plane is a measure of the relative beam intensity across the transverse dimensions of the beam.

Low-mass materials are desirable for profile monitor SEM's. Three factors motivate the use of low- $Z$ materials. First, the number of interaction lengths of material placed in the beam results in beam loss, and such losses cause increased activation of accelerator components and also of surrounding ground water. Second, the interaction of beam particles in the SEM materials causes heating of the device which results in thermal expansion of the 
material and loss of alignment of the strips/wires. Third, thermally-induced dynamic stress in the SEM material may be responsible for signal degradation.

This note procedes as follows: Section 2 discusses the method of the calculation of beam heating used in this note. Section 3 studies the relative beam heating expected as a function of thickness of the SEM material in the beam, and also as a function of the SEM candidate materials. Section 4 uses the expected temperature rise to predict the elongation and tension loss in the SEM planes. Section 5 again uses the expected temperature rise to estimate the temperature stress. Finally in section 6 results of our simulations for beam lines other than NuMI, namely an $8 \mathrm{GeV}$ line or $400 \mathrm{MeV}$ line, where not only the beam energies but also transverse size and per-pulse intensity differ.

\section{Method}

The temperature rise of the SEM's in the beam arises because the power input to each plane from beam energy loss in the material exceeds the power dissipated by conduction of heat to the ends of the strips/wires and the power dissipated via blackbody radiation by the SEM to the walls of the beamline vacuum chamber. The temperature rise of the SEM is given by

$$
m C_{p} \Delta T=\Delta Q=P_{n e t} \Delta t
$$

where $m$ is the mass of a SEM plane, $C_{p}$ the heat capacity of the SEM material in the beam, $\Delta T$ is the temperature rise, $\Delta Q$ is the net energy input within a time increment $\Delta t$, which is given by $\Delta Q=P_{n e t} \Delta t$, with the net power input to the SEM given by:

$$
P_{\text {net }}=P_{\text {beam }}-P_{\text {blackbody }}-P_{\text {conduction }}
$$

In this calculation convective cooling and conduction of heat through the air are neglected since the accelerator pipe is evacuated to $10^{-8}$ Torr.

The beam heating, and consequently the cooling power as well, vary greatly over the surface of the SEM plane. Consequently, we consider the center-most strip or wire in the SEM plane, where the gaussian-peaked beam strikes with greatest intensity. Furthermore, the $12 \mathrm{~cm}$ long strip/wire is divided into 1000 cells along its length, and the above heat balance equation applied separately to each cell. This finite element calculation is carried out for multiple successive time increments $\Delta t=10 \mu \mathrm{sec}$ until thermal equilibrium is achieved. It is assumed that the vacuum chamber walls and also the ends of the SEM strips/wires are held at $T_{\text {ambient }}=25^{\circ} \mathrm{C}$. A view of a single SEM strip/wire is shown in Figure 1.

In all our simulations, we considered a constant $d x=200 \mu \mathrm{m}$ for foil strips, while $d x$ and $d z$ are given by the wire radius $r_{w i r e}$, which will be a varied parameter in our simulations. In the case of foil strips, only the thickness $d z$ is varied. The cell length $\Delta y=0.12 \mathrm{~mm}$ is the same in all simulations. 

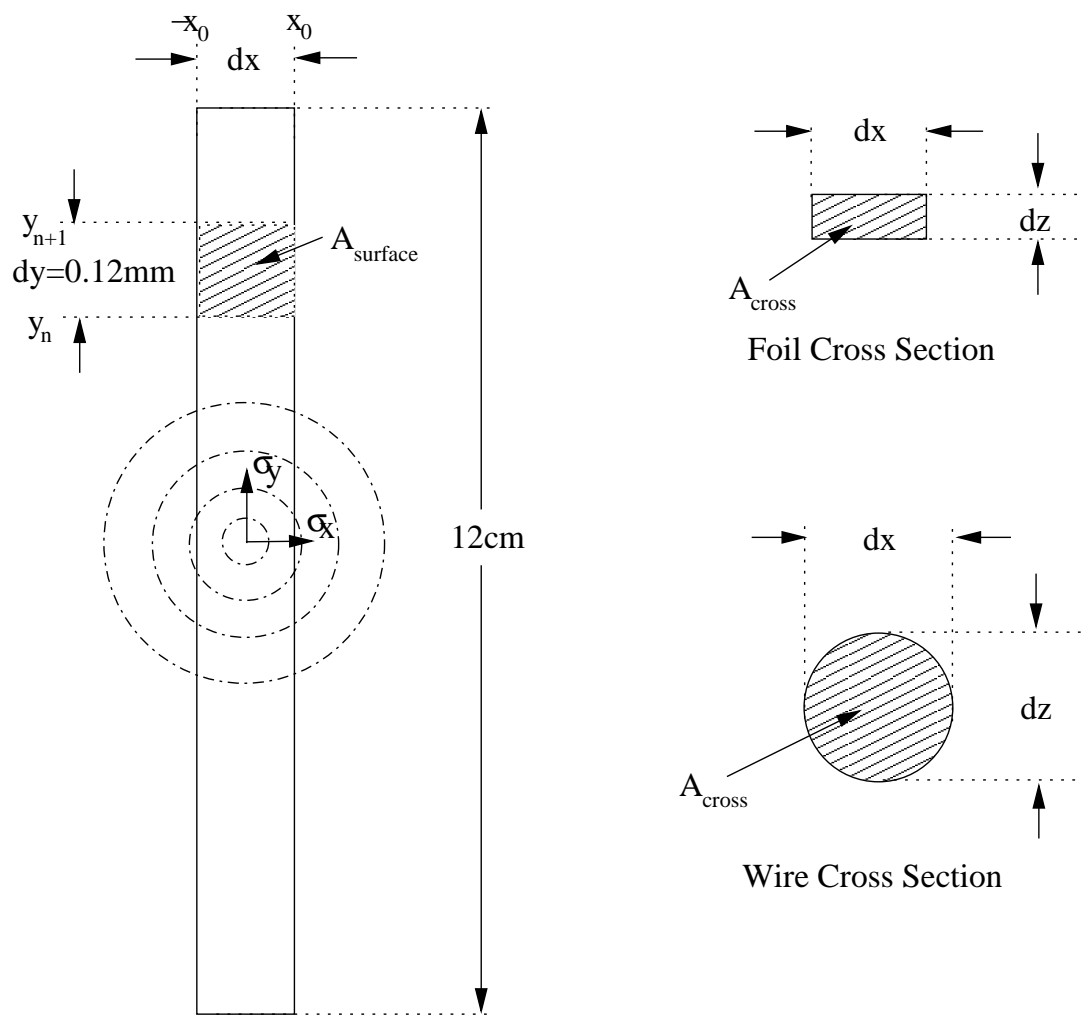

Foil Cross Section

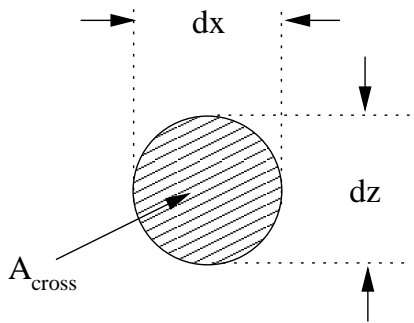

Wire Cross Section

Foil/Wire Top View

Figure 1: Foil/Wire Dimensions and Variables. (left) Beam's eye view of one SEM strip or wire, indicating the beam spot superimposed, and the definition of one cell along the length of the strip/wire. (right) top or cross sectional view of the SEM strip or wire. The $z$ coordinate runs along the beam direction, and $x$ and $y$ are transverse to the beam. The $y$ coordinate runs along the strip/wire length, with $y=0$ at one end of the $12 \mathrm{~cm}$ long strip. The $x$ coordinate is perpendicular to the strip length, with $x=0$ at the center of the strip width. For a wire, $d x=r_{\text {wire }}$ is varied, while for a strip $d x=200 \mu \mathrm{m}$ is assumed throughout.

\subsection{Beam Heating}

The energy deposited by the beam is calculated from the energy loss by ionization expected for a $120 \mathrm{GeV}$ proton. However, using just Bethe-Bloch formula to calculate the proton energy loss and then taking that as a deposited energy would be an over estimate. For thin foils and wires, energetic $\delta$-rays or "knock-on" electrons, which are liberated in the SEM may escape altogether, so that the energy lost by the beam particles through $d E / d x$ may not all be absorbed in the foil. For this reason we use the restricted energy loss formula to 


\begin{tabular}{|c|c|c|c|c|c|}
\hline Material & $-\frac{d E}{d x}\left[\frac{M e V}{g c m^{-2}}\right]$ & $T_{c u t}[M e V]$ & $-\left(\frac{d E}{d x}\right)_{r}\left[\frac{M e V}{g c m^{-2}}\right]$ & $T_{c u t}[M e V]$ & $-\left(\frac{d E}{d x}\right)_{r}\left[\frac{M e V}{g c m^{-2}}\right]$ \\
\hline \hline Beryllium & 2.03 & 0.013 & 1.14 & 0.05 & 1.23 \\
Carbon & 2.26 & 0.014 & 1.26 & 0.05 & 1.37 \\
Aluminum & 2.16 & 0.015 & 1.22 & 0.055 & 1.32 \\
Titanium & 2.02 & 0.018 & 1.14 & 0.07 & 1.23 \\
Nickel & 2.04 & 0.027 & 1.15 & 0.11 & 1.25 \\
Silver & 1.86 & 0.027 & 1.05 & 0.11 & 1.13 \\
Tungsten & 1.67 & 0.033 & 0.94 & 0.14 & 1.02 \\
Gold & 1.67 & 0.033 & 0.93 & 0.14 & 1.01 \\
\hline
\end{tabular}

Table 1: Energy loss of $120 \mathrm{GeV}$ protons computed using the Bethe-Bloch formula and the restricted energy loss using $T_{c u t}$ for $5 \mu \mathrm{m}$ thick foil and $D=50 \mu \mathrm{m}$ wire.

estimate the energy deposited by the proton inside the SEM:

$$
-\left(\frac{d E}{d z}\right)_{T<T_{\text {cut }}}=K z^{2} \frac{Z}{A} \frac{1}{\beta^{2}}\left[\frac{1}{2} \ln \frac{2 m_{e} c^{2} \beta^{2} \gamma^{2} T_{c u t}}{I^{2}}-\frac{\beta^{2}}{2}\left(1+\frac{T_{c u t}}{T_{\max }}\right)-\frac{\delta}{2}\right]
$$

Here the energy transfer is restricted to kinetic energies that are less than some $T_{c u t}$. Details of calculating restricted energy loss are given in appendix A. To estimate $T_{\text {cut }}$ we use tabulated range data for electrons [8], and select $T_{c u t}$ to be the kinetic energy of an electron whose range is equal to half the foil thickness (or equal to the wire radius). Comparison of restricted energy loss to standard Bethe-Bloch result is given for $5 \mu \mathrm{m}$ thick foil and for the wire with diameter $D=50 \mu m$ in table 1 .

To find the total energy deposited by the beam passing through some segment of SEM, we need to find the number of protons passing through that segment. For this we assume that the proton beam has gaussian cross section with similar dimensions in both transvers directions, $\sigma_{x} \approx \sigma_{y} \approx 1 \mathrm{~mm}$.

The number of protons passing through area $A=w \Delta y$ around the point $x=X$ and $y=Y$, is given with:

$$
N_{p}=N_{t o t} \frac{1}{\sqrt{2 \pi} \sigma} \int_{X-w / 2}^{X+w / 2} e^{-x^{2} / 2 \sigma^{2}} d x \frac{1}{\sqrt{2 \pi} \sigma} \int_{Y-\Delta y / 2}^{Y+\Delta y / 2} e^{-y^{2} / 2 \sigma^{2}} d y
$$

where $w$ is the width of the wire/strip and $\Delta y=0.12 \mathrm{~mm}$ is the cell length. If the $\sigma$ of the beam is such that $\sigma>>w$ and $\sigma>>\Delta y$ then we can approximately write:

$$
N_{p} \approx N_{t o t} \frac{1}{\sqrt{2 \pi} \sigma} e^{-X^{2} / 2 \sigma^{2}} w \frac{1}{\sqrt{2 \pi} \sigma} e^{-Y^{2} / 2 \sigma^{2}} \Delta y
$$

For our analysis we want to consider the foil or a wire that is directly in front of the beam so we can look around the $\mathrm{X}=0$ point and finally write:

$$
N_{p} \approx N_{t o t} \frac{w \Delta y}{2 \pi \sigma^{2}} e^{-y^{2} / 2 \sigma^{2}}
$$


Having the number of the protons passing through the segment and the energy deposited by each proton we can easily find the increase of the temperature by equating this total deposited energy to $m C_{p} \Delta T$ :

$$
\begin{gathered}
\Delta Q=m C_{p} \Delta T=N_{p} \rho \frac{d E}{d z} d \bar{z} \\
\Delta T=\frac{N_{p} \rho \frac{d E}{d z} d \bar{z}}{\rho V C_{p}}
\end{gathered}
$$

where $\mathrm{V}$ is the volume of the cell equal to the area of the cross section $A_{\text {cross }}$ times the cell length $\Delta y$ and $d \bar{z}$ is the effective thickness of the cell. For the foil this effective thickness is equal to the thickness of the foil $d z$ because all of the protons passing through the segment are passing through the same amount of the material. For the wire the thickness of the wire in the middle is $2 R$ and it drops to 0 as $\mathrm{x}$ goes to $\pm R$. We can easily find the effective thickness $d \bar{z}$ :

$$
d \bar{z}=2 \cdot \int_{-R}^{R} \frac{\sqrt{R^{2}-x^{2}}}{2 R} d x=2 R \frac{\pi}{4}
$$

Now using the equation for number of protons passing through the segment we get:

$$
\Delta T=N_{t o t} e^{-y^{2} / 2 \sigma^{2}} \frac{w \Delta L}{2 \pi \sigma^{2}} \frac{\frac{d E}{d z} d \bar{z}}{V c_{p}}
$$

For the foil, the volume of the cell $V=w d z \Delta y$ cancels out of temperature rise. The same is true for a wire, since $w \Delta y d \bar{z}=(2 R)(\Delta y)\left(2 R \frac{\pi}{4}\right)=V$

$$
\Delta T=\frac{N_{t o t} e^{-y^{2} / 2 \sigma^{2}}}{2 \pi \sigma^{2} C_{p}} \frac{d E}{d z}
$$

A weak volume dependence in $\Delta T$ is implicit in Equation 9 due to the restricted energy loss $\frac{d E}{d z}$, which depends on $T_{c u t}$. This dependence is somewhat different for foils and wires.

\subsection{Heat Simulation}

We need to consider the problem in which the SEM is being periodicaly heated by beam pulses. Cooling between the pulses is predominately due to heat conduction and blackbody radiation. We can write down the differential equation governing the cooling that includes those two terms:

$$
\frac{\partial T}{\partial t}=\alpha \nabla^{2} T-\frac{\sigma\left(T^{4}-T_{a m b i e n t}^{4}\right)}{c_{p} \rho V} \epsilon A_{\text {surf }}
$$

where $\epsilon$ is the surface emissivity, $A_{\text {surf }}$ is surface area and $\alpha$ is thermal diffusivity given with:

$$
\alpha=\frac{k}{C_{p} \rho}
$$




\begin{tabular}{|c|c|c|c|c|c|c|}
\hline Material & $Z$ & $\begin{array}{c}\text { Density } \\
\left(\mathrm{g} / \mathrm{cm}^{3}\right) \\
{[3]}\end{array}$ & $\begin{array}{c}\text { Heat } \\
\text { Capacity } \\
\left(\mathrm{J} / \mathrm{g}^{\circ} \mathrm{C}\right)[3]\end{array}$ & $\begin{array}{c}\text { Thermal } \\
\text { Conductivity } \\
\left(\mathrm{W} / \mathrm{cm}^{\circ} \mathrm{C}\right)[3]\end{array}$ & $\begin{array}{c}\text { Emissivity } \\
\text { in Simulation }\end{array}$ & $\begin{array}{c}\text { Emissivity } \\
{[3]}\end{array}$ \\
\hline Beryllium & 4 & 1.848 & 1.82 & 2.18 & 0.1 & - \\
\hline Carbon & 6 & 2.265 & 0.690 & 0.24 & 0.8 & $0.7-0.8^{a}$ \\
\hline Aluminum & 13 & 2.70 & 0.899 & 2.37 & 0.1 & $0.04-0.06$ \\
\hline Titanium & 22 & 4.54 & 0.523 & 0.2 & 0.2 & - \\
\hline Nickel & 28 & 8.902 & 0.443 & 0.899 & 0.1 & $0.07-0.09$ \\
\hline Silver & 47 & 10.5 & 0.238 & 4.27 & 0.1 & $0.02-0.04$ \\
\hline Tungsten & 74 & 19.3 & 0.134 & 1.78 & 0.1 & $0.02-0.07^{b}$ \\
\hline Gold & 79 & 19.32 & 0.130 & 3.15 & 0.1 & 0.03 \\
\hline$a$ graphite & & \multicolumn{7}{c}{${ }^{b}$ for temperatures $<500^{\circ} \mathrm{C}$} &
\end{tabular}

Table 2: Properties of candidate SEM materials which enter the calculation of the temperature rise due to beam heating.

$k$ is thermal conductivity.

Given the number of protons and the beam profile we can calculate the increase in temperature for each cell along the wire or foil strip. This then gives us initial conditions. On the boundaries the SEM is on ambient temperature. With these boundary and initial conditions we can numericaly solve the differential eqaution for the period of cooling. Then we can again add the heat from the beam and repeat calculation with new initial conditions. We can repeat this until we get to the desired number of proton pulses.

To solve the equation numericaly we need to divide a foil or a wire of length $\mathrm{L}$ into $\mathrm{N}$ cells. For the i-th cell we can then write:

$$
\frac{\partial T_{i}}{\partial t}=\frac{\alpha}{(\Delta y)^{2}}\left(T_{i+1}-2 T_{i}+T_{i-1}\right)-\frac{\sigma\left(T_{i}^{4}-T_{a m b i e n t}^{4}\right)}{C_{p} \rho V} \epsilon A_{s u r f}
$$

Our initial conditions for proton pulse $n_{p}$ are:

$$
\begin{gathered}
T_{i}^{n_{p}}=T_{i}^{n_{p}-1}+\Delta T_{i} \\
T_{i}^{n_{p}}=T_{i}^{n_{p}-1}+\frac{N_{t o t} e^{-\left(\frac{L}{2}-i \Delta y\right)^{2} / 2 \sigma^{2}}}{2 \pi \sigma^{2} C_{p}} \frac{d E}{d z}
\end{gathered}
$$

with $T_{i}^{n_{p}-1}$ being the temperature of the i-th segment just before the next proton pulse and $T_{i}^{0}=T_{\text {ambient }}$. The boundary conditions are:

$$
T_{0}=T_{N}=T_{\text {ambient }}
$$

Various parameters for different materials that were considered are listed in Table 2.

As was mentioned before for this simulation we choose to divide the SEM into 1000 cells, so for a $12 \mathrm{~cm}$ long SEM $\Delta y=0.012 \mathrm{~cm}$, and for a time increment we choose $\Delta t=10 \mu \mathrm{s}$. This value was chosen because smaller time scales greatly increase CPU time to unproductive levels. Yet, from Figure 2, one can see that the equilibrium temperature of the foil at its center approaches an asymptotic value for very small time increments $\Delta t$ used in the 


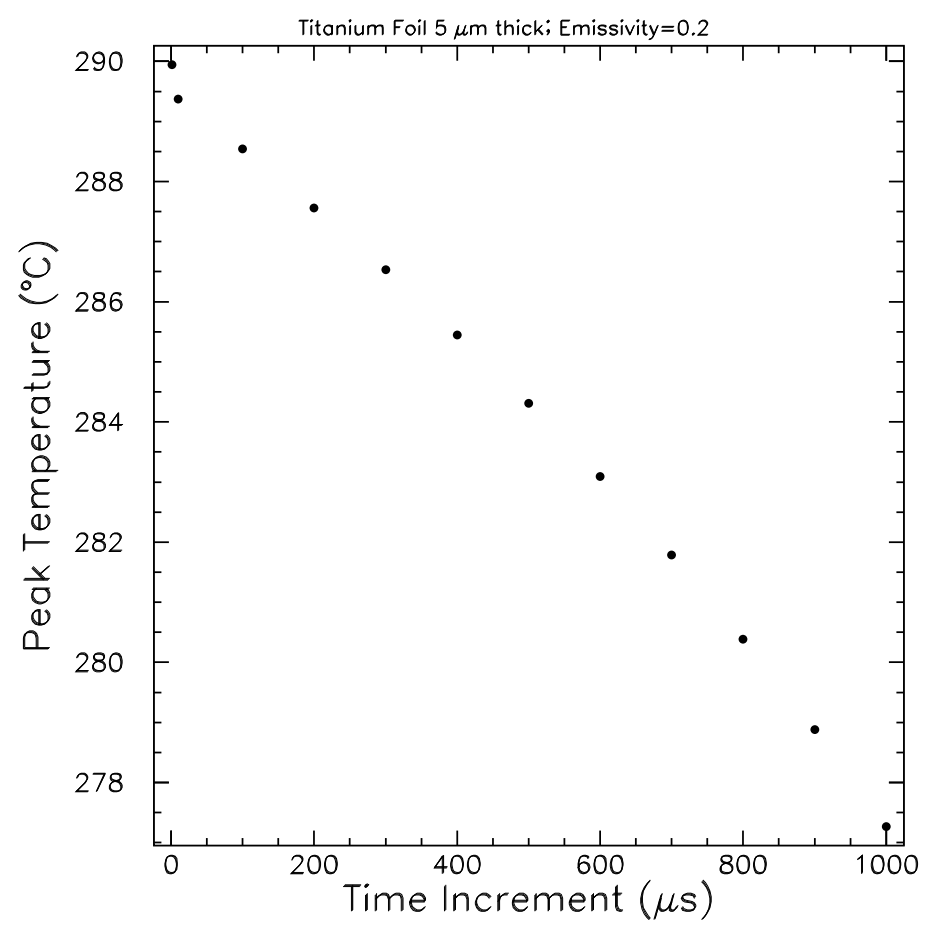

Figure 2: Equilibrium temperature of the center of a SEM strip plotted as a function of the time increment $\Delta t$ used in the finite element simulation. Note the suppressed zero.

simulation. This variation observed in Figure 2 is due in part to our desire to use very small cell sizes and in part to the fact that the heat due to condution can travel all the way across one cell distance $\Delta y$ in one time increment if $\Delta t$ is too long. Figure 2 suggests that the error in the temperature calculations is of the order of a couple of percent due to the choice of $\Delta t=10 \mu \mathrm{sec}$. In calculations where time increment was less then the beam spill time, the beam heating was distributed throughout the first $n=\frac{\text { beam spill time }}{\Delta t}$ time increments.

Another motivation to choose a particular $\Delta t$ comes from the numerical stability condition for heat conduction equation. The stability condition is:

$$
\alpha \frac{\Delta t}{\Delta y^{2}}<\frac{1}{2}
$$

Therefore, since $\alpha<2 \mathrm{~cm}^{2} / s$ for all the materials considered, this is satisfied with our choice of $\Delta t$ and $\Delta y$.

During one beam cycle, the impinging beam causes a sharp temperature rise. Following this rise, blackbody radiation and heat conduction allow the foil to cool. This heating and cooling process is evident in Figure 3, which shows the time-development of the temperature at the central cell of a foil strip. The foil is largely able to cool itself within the 1.9 sec beam cycle, leading in this case to temperature cycles of $220^{\circ} \mathrm{C}$. Thermal equilibrium, of sorts, is 


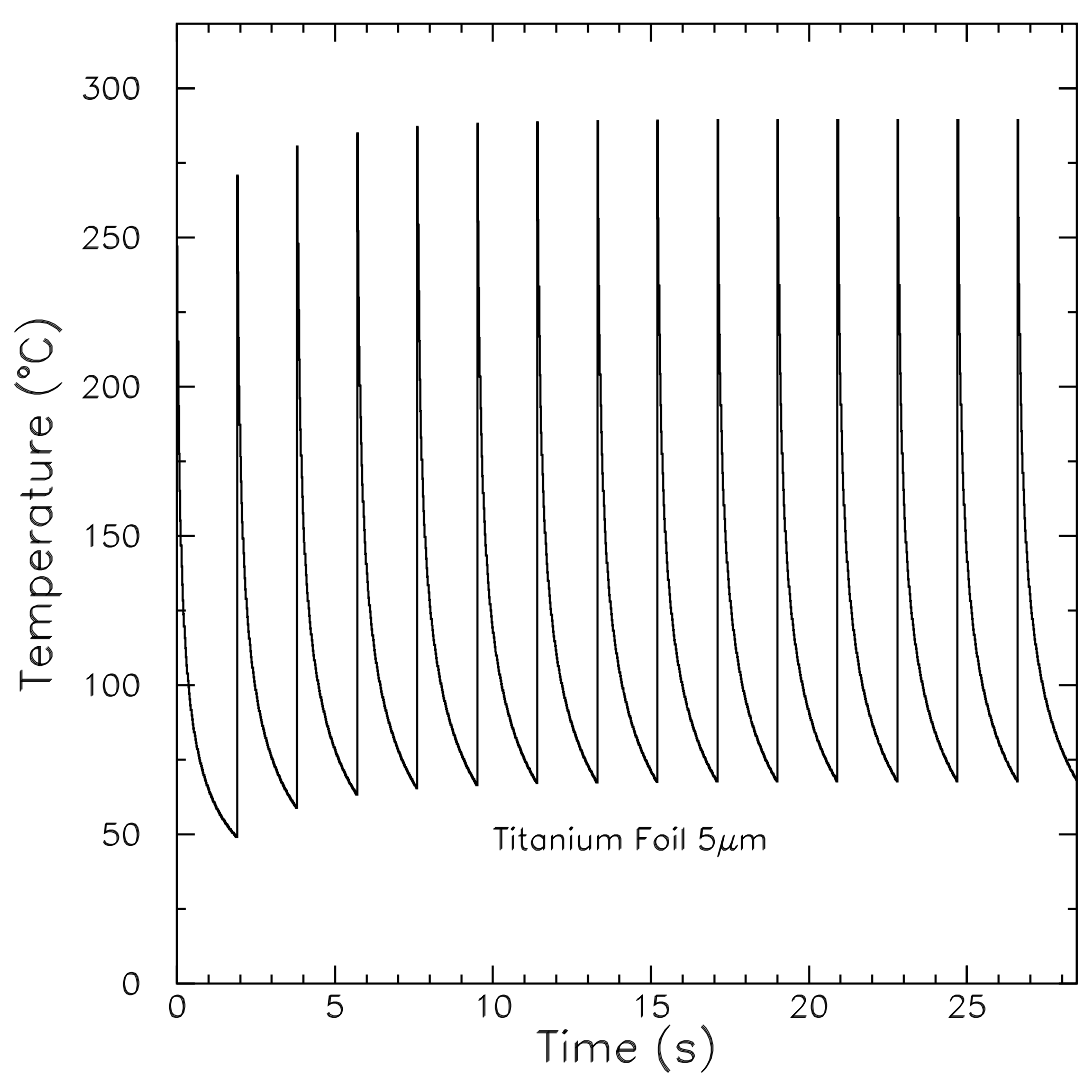

Figure 3: Temperature of the center cell of a $5 \mu \mathrm{m}$ thick Titanium foil as a function of time. The repeated beam cycles each $1.9 \mathrm{sec}$ may be seen as evidenced by the sharp temperature rise.

achieved within 15 sec.

The time-evolution of the temperature along the foil's length is shown in Figure 4. Shown is the temperature as a function of position along the length of the strip. Several snapshots over the course of one beam cycle are plotted. The figure is made for the beam cycle which occurs at $t=26.6 \mathrm{sec}$ in Figure 3. As can be seen, the beam causes a sharp peak in the temperature profile with $\sigma_{y} \approx \sigma_{\text {beam }}=1 \mathrm{~mm}$. Later in time, the heat is dissipated by blackbody radiation, reducing the temperature. The heat also flows via thermal conduction to the ends of the strip. This flow is evidenced by the widening peak in the temperature profile, caused by the flow of heat from the center of the strip at $y=6 \mathrm{~cm}$ out to the edges which are held at $T_{\text {ambient }}=25^{\circ} \mathrm{C}$. A similar calculation is shown for a $50 \mu \mathrm{m}$ diameter $\mathrm{Ti}$ wire in Figure 5.

Figure 6 shows the results of a simulation which justifies our statement that blackbody 


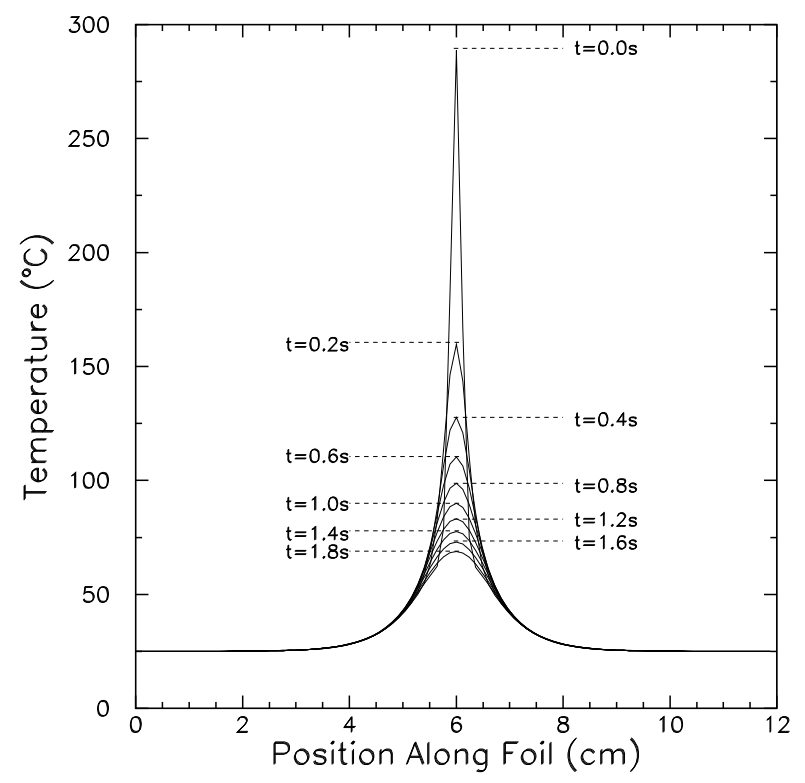

Figure 4: Cooling of a foil from its peak temperature during one beam spill. The beam cycle simulated here occurs at $t=26.6$ sec in the simulation shown in Figure 3.

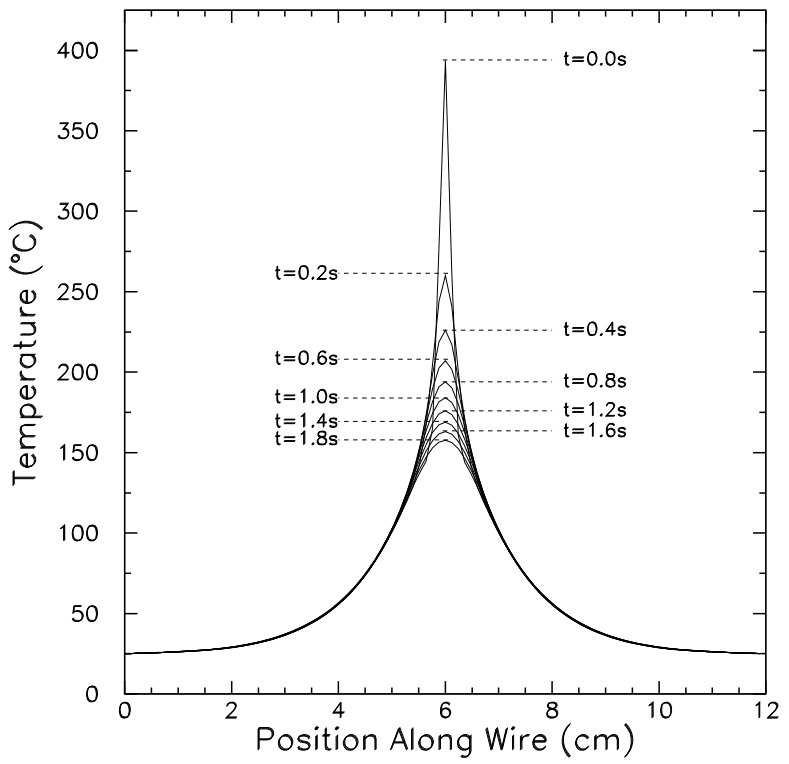

Figure 5: Cooling of a wire from its peak temperature. The simulation is similar to that shown in Figure 4, but is shown here for a $50 \mu \mathrm{m}$ diameter $\mathrm{Ti}$ wire. 


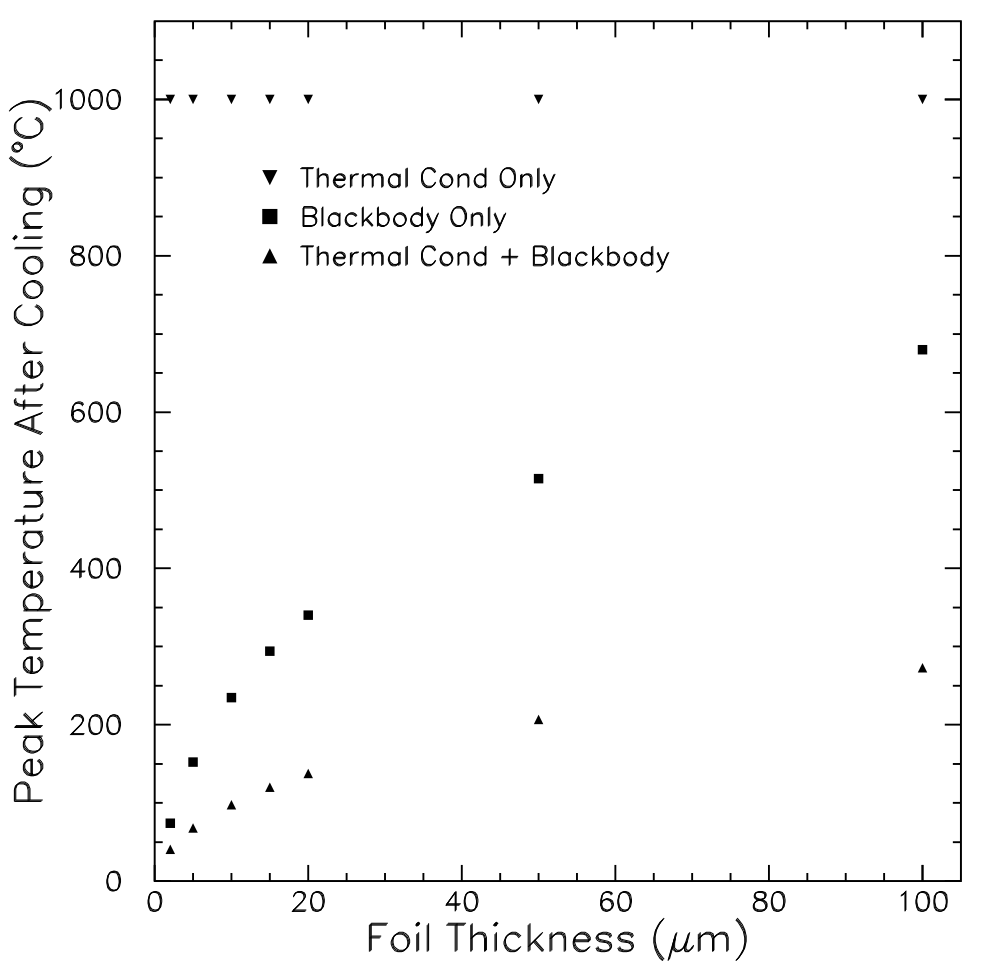

Figure 6: Comparison of different cooling mechanisms on the equilibrium temperature. Shown is the result of a simulation in which beam heating is compensated by either blackbody radiation, by thermal conduction through the strip, or by both these effects.

radiation is the dominant cooling mechanism for all but the thickest SEM's. The simulation tracks the peak temperature at the center of a foil strip as a function of the thickness of the foil, and the temperature is plotted at equilibrium, immediately before the next beam spill occurs. In this simulation, either blackbody radiation or thermal conduction through the material is successively turned off. It may be seen that for very thin foils, where the surface-to-volume ratio is large, blackbody radiation is clearly more efficient at cooling the foil. Only as the foil thickness grows is the effect of turning off conduction particularly strong. Given that blackbody radiation does dominate, our bias is to consider thin $(2-5 \mu \mathrm{m})$ foils. 


\section{Alternative SEM Configurations}

\subsection{Variation of Thickness}

The simulations of Titanium wire and foil in Section 2 considered only $5 \mu \mathrm{m}$ thick foil and $50 \mu \mathrm{m}$ diameter wire. Thinner foils are possible, albeit with less regularity in the thickness. At $2.5 \mu \mathrm{m}$ thickness, for example, the variation will be nearly $20 \%$. Thicker foils are readily available as well, and are in general easier to handle. The chemical etching process is easier with thicker parts, although the etching definition is less sharp.

Figures 7 and 8 show the behaviour of the SEM temperature at the center of a strip/wire as a function of the thickness (foil) or diameter (wire). In each figure are plotted the temperatures in the time increment just after the beam spill has occurred, and also for the time increment just prior to the next beam spill. The values for temperatures are taken from the beam cycle when the "equilibrium" was already reached, so the difference between the peak temperature just after the beam spill and just before the next beam spill is equal to $\Delta T$. From Equation 9 we see that $\Delta T$ explicitly depends only on energy deposited in the material and not on the shape of the material, but as was argued before the energy deposited depends on the shape of the SEM through $T_{\text {cut }}$.

The calculations are performed for several possible values of the emissivities, since this quantity is not well known for Titanium (for shiny Aluminum or Gold, the CRC reports a reasonably well-known value of 0.1 , but Titanium can be a fair bit 'duller'). Our previous calculations, for example, assumed $\epsilon=0.2$.

\subsection{Variation of Material}

In addition to Titanium, other low- $Z$ candidates for either foil or wire SEM's include Be, $\mathrm{Al}$, or C. Beryllium is challenging because of it bio-toxicity. Aluminum is non-ideal because its secondary electron emission signal degrades significantly with beam exposure [2]. However, Carbon filaments have been used by workers at SLAC and Los Alamos [9]. A $30 \mu \mathrm{m}$ Carbon filament, for example, has about the same beam loss as the $5 \mu \mathrm{m}$ Titanium foil.

The results of a simulation using different materials is shown in Figure 9. In general, the plot shows the expected rise of foil center temperature with atomic number $Z$, which is expected due to the increased energy loss by ionization by beam particles in the material (see Table 2). Small variations about this trend, however, are visible. For example, Carbon, while it has an increased temperature just after the spill relative to Beryllium, has a quite low temperature after the $1.9 \mathrm{sec}$ before the next beam spill. This is because it has a large emissivity $\epsilon \sim 0.8$. Aluminum appears to drop below the trend in heating $v s$. $Z$, which may well be due to its large thermal conductivity and heat capacity relative to other materials considered (see Table 2). 

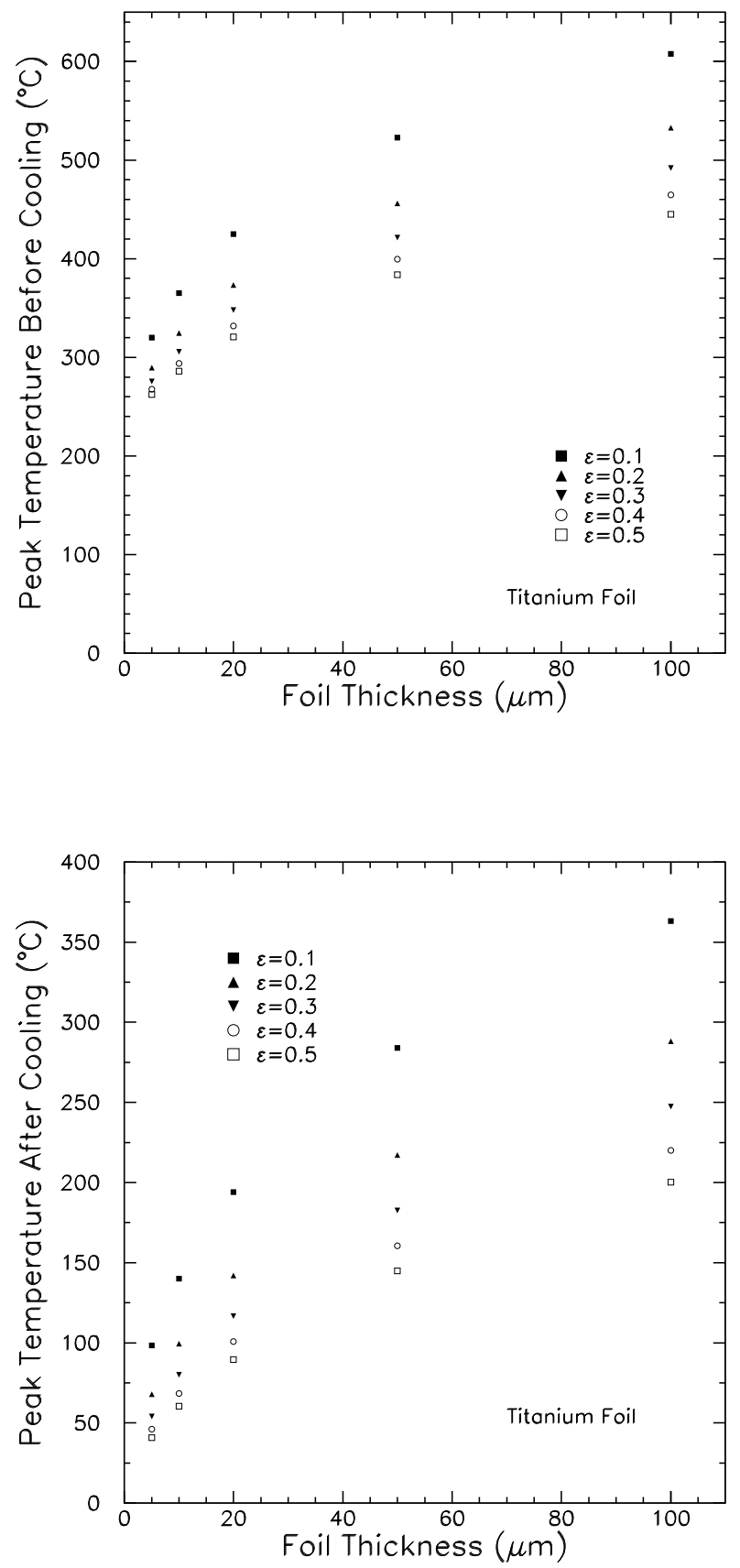

Figure 7: Peak temperature at the center of a Ti foil, shown for several possible emissivities $\epsilon=0.1-0.5$ and several foil thicknesses. (above) Temperature just after a beam spill (below) Temperature just prior to the next beam spill. 

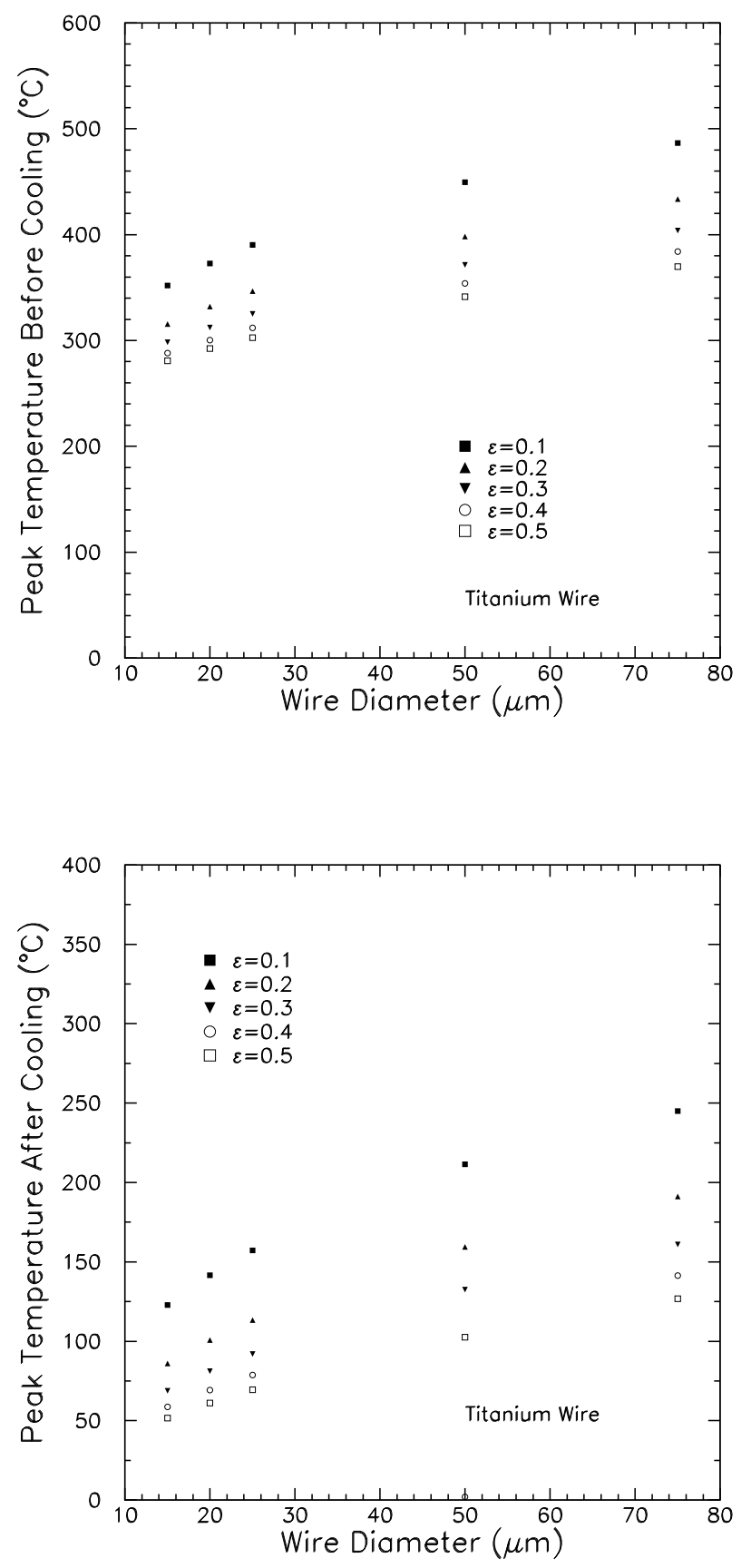

Figure 8: Peak temperature at the center of a Ti wire, shown for several possible emissivities $\epsilon=0.1-0.5$ and several wire diameters. (above) Temperature just after a beam spill (below) Temperature just prior to the next beam spill. 

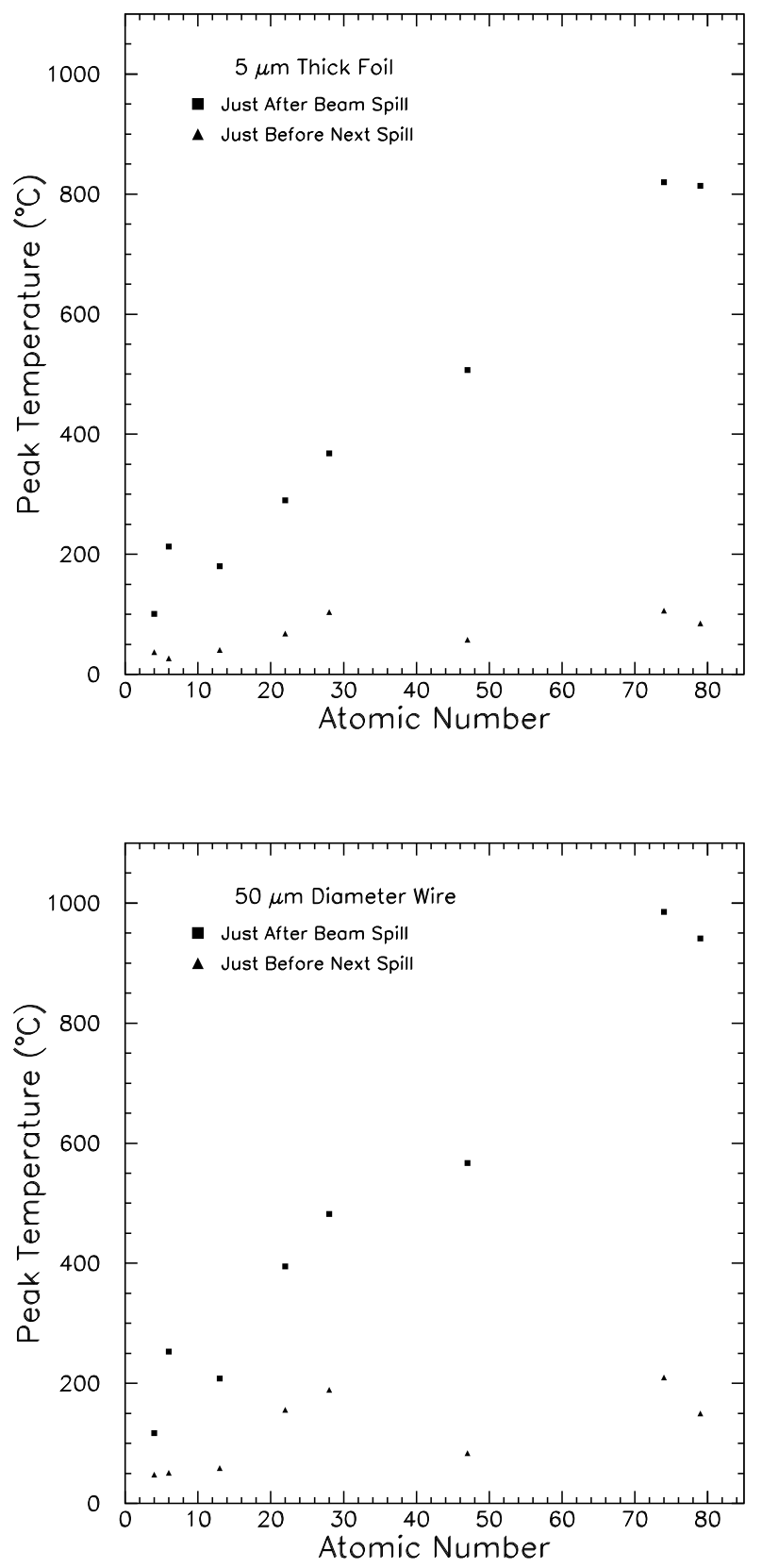

Figure 9: Peak temperature at the center of a strip vs atomic number for $5 \mu \mathrm{m}$ thick foil (above) and $50 \mu \mathrm{m}$ diameter wire (below) Shown are the peak temperatures just after and just before a beam spill. 


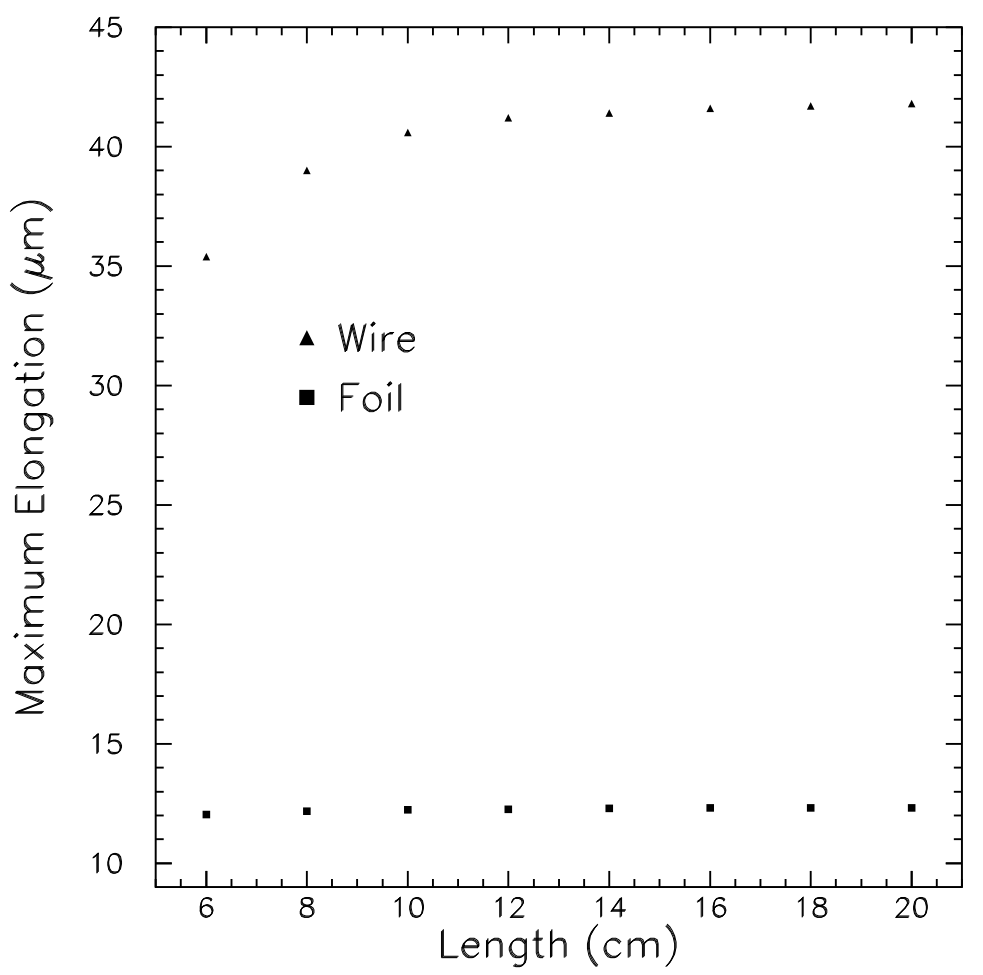

Figure 10: Thermal expansion of a $50 \mu \mathrm{m}$ diameter $\mathrm{Ti}$ wire and a $5 \mu \mathrm{m}$ thick $\mathrm{Ti}$ foil as a function of the length of the strip/wire. Note the suppressed zero.

\subsection{Variation of Length}

The length of the strip or wire does not greatly affect the peak temperature at the center of the strip/wire. It does, however, affect the overall elongation of the strip/wire because heat which fails to flow out to the ends of the segment will result in temperature build-up in the "tails" of the peak. This build-up may be noted as the broad gaussian-like profile in Figures 4 and 5 . The effect of varying the length is of greater importance for wires, which do benefit by thermal conduction, than it is for thin foils, which dominately cool through blackbody radiation.

In Figure 10 we study the effect of the length of the strip or wire for a Titanium SEM (either $50 \mu \mathrm{m}$ diameter wire or $5 \mu \mathrm{m}$ thick foil). We plot the net elongation (see next section) due to thermal expansion of the strip/wire vs. its length. Note that previous simulations have been performed with $L=12 \mathrm{~cm}$. 


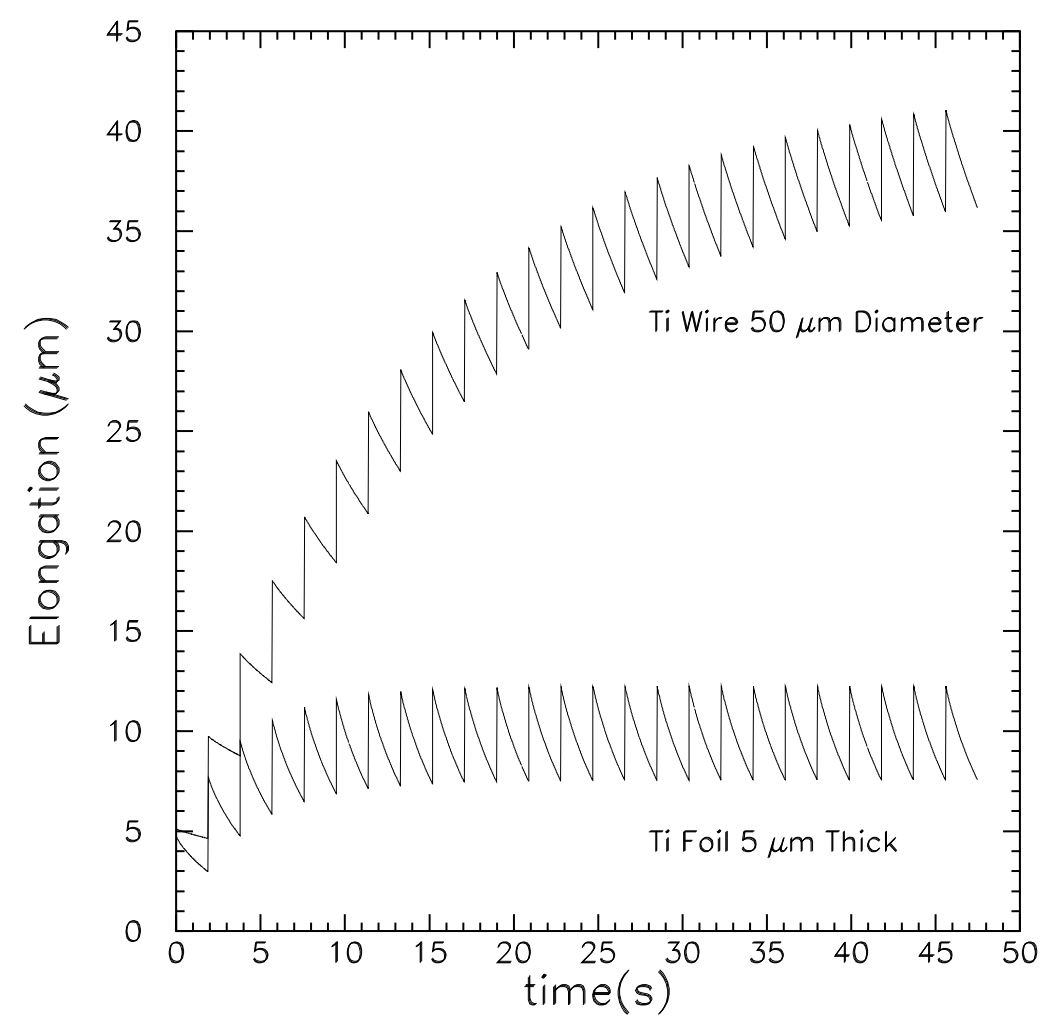

Figure 11: Elongation and Contraction vs. time for $120 \mathrm{~mm}$ long Titanium $50 \mu \mathrm{m}$ diameter wire and $5 \mu \mathrm{m}$ thick foil.

\section{Elongation and Tension Loss}

The temperature profiles calculated for the many scenarios considered above have been used as the input for the calculation of how much linear expansion is expected due to the net beam heating. The coefficients of thermal expansion for the various materials considered (see Table 3) is multiplied by the cell size $d y$ and the temperature rise from our thermal calculations, and the result summed over the entire length of a strip or wire. Results of calculated linear expansion are shown in Figures 11, 13, and 14.

The results of Figure 11 indicate that the elongation of a $5 \mu \mathrm{m}$ thick foil SEM will result in negligible effects on the strip tension. Foil SEM's, as is discussed in a separate note [12], may be manufactured with accordion-like "springs" pressed into the two ends of the strips. These spring-loaded foils may be pulled over a frame in such a way as to expand by 6-10 mm in the process of stretching the foil SEM on the frame. The resulting tension is of order a couple of grams, sufficient to limit gravitational sag $<100 \mu \mathrm{m}$. A schematic sketch of the accordion spring concept is shown in Figure 12. 


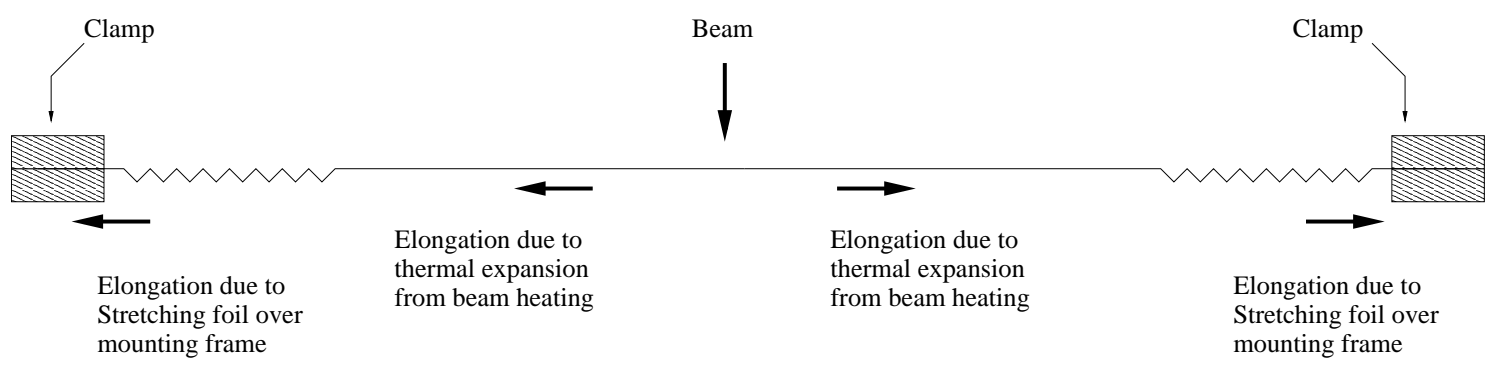

Figure 12: Schematic sketch of the accordion springs to be pressed into the foil. The accordion springs are stretched outward by $6-10 \mathrm{~mm}$ when the foil is mounted on the frame. Beam heating causes thermal expansion of the foil from the center, which relaxes some of the tension built up in the springs.

Inspection of Figure 14 shows that for all materials, a foil SEM has maximum elongation due to beam heating of at most $160 \mu \mathrm{m}$. For our preferred Titanium foil design, the maximum elongaton is a modest $12 \mu \mathrm{m}$. This elongation of $12 \mu \mathrm{m}$ is less then $1 \%$ of the elongation used to tension the foil SEM. Thus we conclude that the tension loss of the Titanium foil SEM is of order less then a percent.

The elongation of the SEM wires, in contrast, can result in tension loss. We attempt to estimate this tension loss using the estimated elongation of the wires from Figure 14, assuming that the wires are strung over a frame as is done in conventional wire chamber design, using an applied tension to limit wire sag.

The definition of the Young's modulus is given by

$$
E=\frac{L_{0}}{\Delta L_{0}} \frac{F}{A}
$$

where $E$ is the Young's modulus (in Pascales), $L_{0}$ is the original or equilibrium strip/wire length, $F$ is the applied force (in Newtons), $A$ is the cross sectional area of the strip or wire, and $\Delta L_{0}$ is the elongation of the strip or wire which results from this force $F$. The linear expansion continues as long as $F$ is less than the yield strength (in $\mathrm{Pa}$ ) of the material, which is the applied stress under which plastic deformation, or permanent distortion of the material, occurs.

As is well known, wire chambers may be strung only up to a maximum applied tension, lest the wires stretch inelastically and break. For the purposes of our discussion, we take this elastic limit to be the Yield Stress. ${ }^{1}$ In Table 3, we have converted Yield Stress to grams of mass that could be used to tension a $50 \mu \mathrm{m}$ diameter wire, just for the reader's reference. At this maximum tension, there is an elongation to be expected in the wire. From the above definition of the Young's modulus, this elongation is

$$
\frac{\Delta L_{\max }}{L_{0}}=\left(\frac{F}{A}\right)_{\max } \frac{1}{E} \leq \frac{Y}{E}
$$

\footnotetext{
${ }^{1}$ The actual elastic limit is typically less.
} 


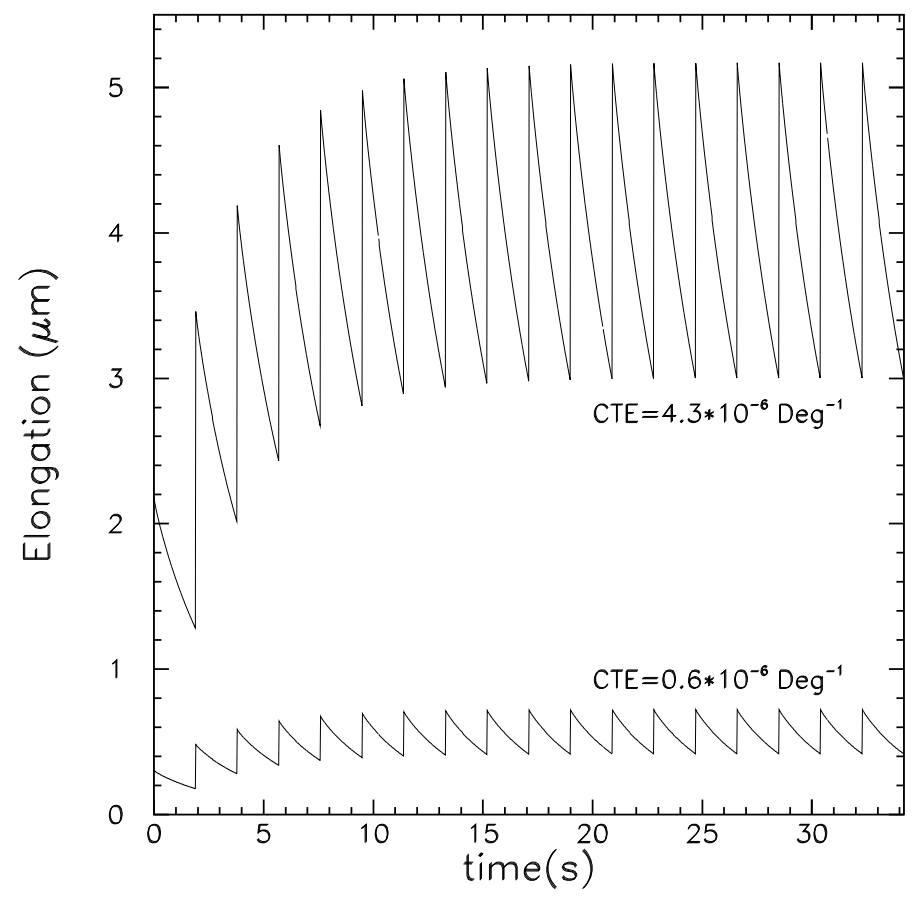

Figure 13: Elongation and Contraction vs. time for $120 \mathrm{~mm}$ long Carbon $50 \mu \mathrm{m}$ diameter wire. Because of the range of accepted values for the CTE of Carbon, the calculation was performed with both the maximum and minimum CTE value.

where $Y$ is defined as the yield stress. This maximum elongagion is tabulated in column 7 of Table 3.

The heating of the wire by the beam results in some loss in tension, or equivalently, a loss in the relative elongation of the wire that is strung over a frame. The beam heating data for $50 \mu \mathrm{m}$ diameter wires in Figure 14, has been tabulated in Table 3. As can be seen, a $50 \mu \mathrm{m}$ Tungsten wire, for example, can lose nearly $40 \%$ of its applied tension. The Aluminum wire loses all of its tension.

The present study suggests that the use of springs on individual wires, employed by several workers in the field of beam instrumentation $[9,10]$, is an important step to ameliorate the effect of beam heating on wire SEM's. Only Carbon wires appear to have a thermal expansion far less than the elongation expected in stringing the wires.

This estimate of tension loss is somewhat over-pessimistic because the elongation of the wire cycles between two values (the maximum just after the beam spill, the minimum just before the next beam spill), and is not equal to the maximum plotted in Figure 14. On the other hand, this estimate underplays the tension loss, since in practice one applies somewhat less than the yield stress to a wire. 


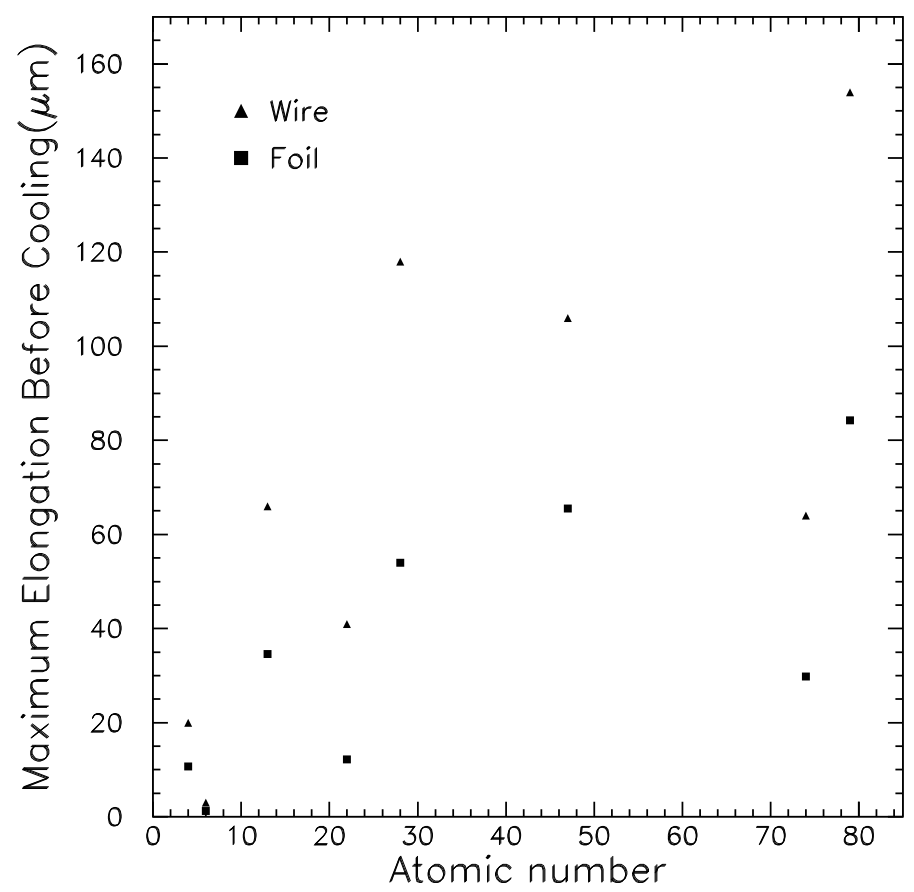

Figure 14: Maximum elongation due to beam heating for strips/wires of $120 \mathrm{~mm}$ original length $v s$. the atomic number of the material.

\begin{tabular}{|c|c|c|c|c|c|c|c|}
\hline Material & $Z$ & $\begin{array}{c}\mathrm{CTE} \\
\left(10^{6} /{ }^{\circ} \mathrm{C}\right)[3]\end{array}$ & $\begin{array}{c}\text { Yield } \\
(\mathrm{MPa})\end{array}$ & $\begin{array}{l}\text { rength [4] } \\
\text { (grams) }^{a}\end{array}$ & $\begin{array}{l}\text { Young's Mod. } \\
(\mathrm{GPa})[4,5]\end{array}$ & $\begin{array}{c}\text { Fractional } \\
\text { Elongation at } \\
\text { Yield Str. }\left(\times 10^{-3}\right)\end{array}$ & $\begin{array}{c}\text { Fractional } \\
\text { Elongation from Beam } \\
\text { Heating }\left(\times 10^{-3}\right)^{a, b}\end{array}$ \\
\hline Beryllium & 4 & 12 & $240^{d}$ & 48 & 287 & 0.84 & 0.17 \\
\hline Carbon & 6 & $0.6-4.3$ & $469^{c}$ & $40-45^{c}$ & $40.3^{c}$ & 11.6 & $0.0067-0.043$ \\
\hline Aluminum & 13 & 25 & $10-35^{d}$ & $2-7$ & 70.3 & $0.14-0.50$ & 0.55 \\
\hline Titanium & 22 & 8.5 & $220^{e}$ & 44 & 115.7 & 1.9 & 0.342 \\
\hline Nickel & 28 & 13 & $1580^{d}$ & 316 & 199.5 & 7.9 & 0.983 \\
\hline Silver & 47 & 19 & - & - & 83 & - & - \\
\hline Tungsten & 74 & 4.5 & $550^{d}$ & 110 & 411 & 1.34 & 0.54 \\
\hline Gold & 79 & 14.2 & $205^{f}$ & 41 & 82.7 & 2.48 & 1.283 \\
\hline
\end{tabular}

Table 3: Properties of candidate SEM wire materials which enter the calculation of mechanical elongation and loss of tension. The CTE $\equiv$ "Coefficient of Thermal Expansion". The yield strength, normally expressed in Pascales, is also translated to the gram-equivalent mass which would break a $50 \mu \mathrm{m}$ diameter wire. The fractional elongation at the maximum applied stress (the yield stress) is $\equiv$ (Yield Stress)/(Young's Mod.) and is the fractional amount the material would stretch elastically before breaking. 


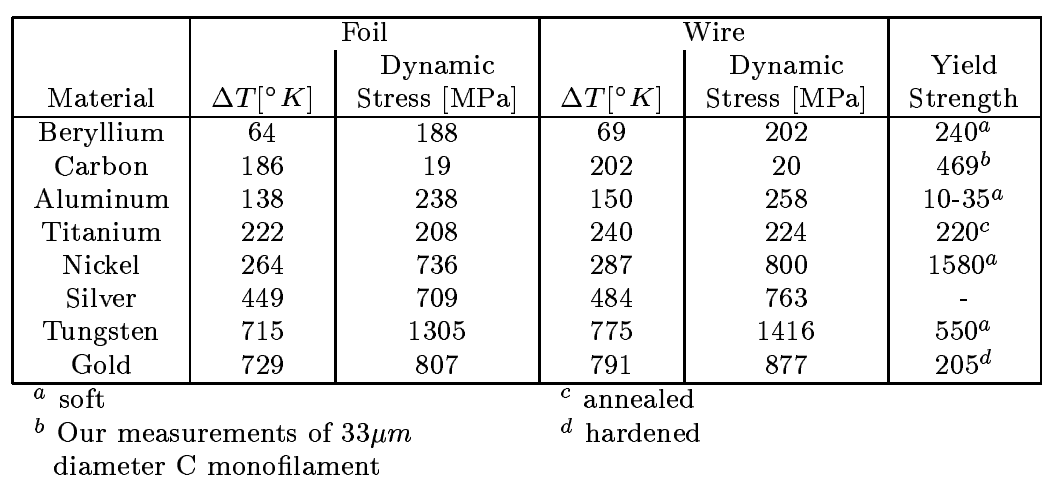

Table 4: Comparison between dynamic stress and yield strength for a $5 \mu m$ thick foil and a $50 \mu m$ diameter wire.

\section{Dynamic stress}

For NuMI, the $8.6 \mu \mathrm{s}$ proton beam spill time is very short on the time scales involved in heat conduction and blackbody radiation and that is the reason why the temperature rise was mostly treated like the initial condition since in most of the calculations the time increment $\Delta t$ was greater than the spill length. This, of course, means that there will be a sharp temperature rise in the SEM. This sudden increase in temperature causes a stress in a material given with [11]:

$$
S=E \alpha \Delta T
$$

where $E$ is the Young's modulus and $\alpha$ is the coefficient of thermal expansion. If this stress is greater than some critical value the material can be deformed or it can even break. As in Section 4 the yield strength can be taken as a comparison.

The maximal stress will be produced in a segment which will heat up the most. Using Equation 9 we can find this $\Delta T$. Tabel 4 lists results for a $5 \mu m$ thick foil and $50 \mu m$ diameter wire made of various materials and compares them to the yield strength. 


\section{Calculations for other Beam lines}

For completeness, we investigate the behavior of a profile monitor SEM in beamlines other than the NuMI beam. The NuMI beam is actually quite an unusual case, because while the cycle time is not particularly fast, the per-pulse intensity is quite high and the spot size is quite small, yielding very high temperature rise and dynamic stress values for most materials.

Other beam lines, such as the $8 \mathrm{GeV}$ transfer line from the Booster to the Main Injector or the $8 \mathrm{GeV}$ transport line to MiniBooNE, or the $400 \mathrm{MeV}$ line, have lower per-pulse intensity but in contrast to NuMI run at quite rapid repetition rates. We list some of the relevant differences between these lines and the NuMI line below.

When simulating a $400 \mathrm{MeV}$ line, we have made an important assumption, namely that the beam consists of $400 \mathrm{MeV}$ kinetic energy protons, not $\mathrm{H}^{-}$ions. There is actually quite an important difference between the two beam species, as the $\mathrm{H}^{-}$ions can strip in the SEM foil material, and the stripped electrons also deposit energy in the foils. In beam lines such as the SNS, the energy deposition from the stopping electrons is over four times the energy deposition of the passing protons [13]. Thus, our naive calculation is appropriate only for an extracted $400 \mathrm{MeV}$ beam, such as might be envisioned for the MuCool project.

\begin{tabular}{|c|c|c|c|}
\hline & $400 \mathrm{MeV}$ linac & MiniBoone & NuMI \\
\hline Beam Kinetic Energy $(\mathrm{GeV})$ & 0.4 & 8 & 120 \\
Spill Length $(\mu \mathrm{s})$ & 25 & 1.56 & 8.6 \\
Rep. Rate $(\mathrm{Hz})$ & 15 & 5 & 0.53 \\
Protons/spill & $5 \times 10^{12}$ & $5 \times 10^{12}$ & $4 \times 10^{13}$ \\
Spot Size, $\sigma(\mathrm{mm})$ & $\sim 5$ & $\sim 2$ & $\sim 1$ \\
\hline
\end{tabular}

Table 5 lists the results for titanium foil, titanium wire and tungsten wire for the three beam lines. Altough the temperatures are quite different for NuMI beam line and Linac, the fractional elongations are almost the same because the temperature distribution along the foil/wire is different. It is much broader for Linac than for NuMI. They are listed below. With these inputs, we estimate the results shown in Figure 15, which shows a graph of the temperature and elongation of a $\mathrm{Ti}$ foil SEM in the $8 \mathrm{GeV}$ MiniBooNE line as a function of time.

Figure 16 shows the temperature distribution along the foil/wire for Linac and MiniBoone. Again, from these plots we can see that cooling of the foil is almost entirely due to blackbody radiation, there is almost no flux of heat to the boundaries. For the wire we see that the peak is broader and there is some heat flux into the boundaries which contributes somewhat to cooling. Further information, including temperature rise, elongation, and restricted $d E / d x$, are given in Table 5 . 

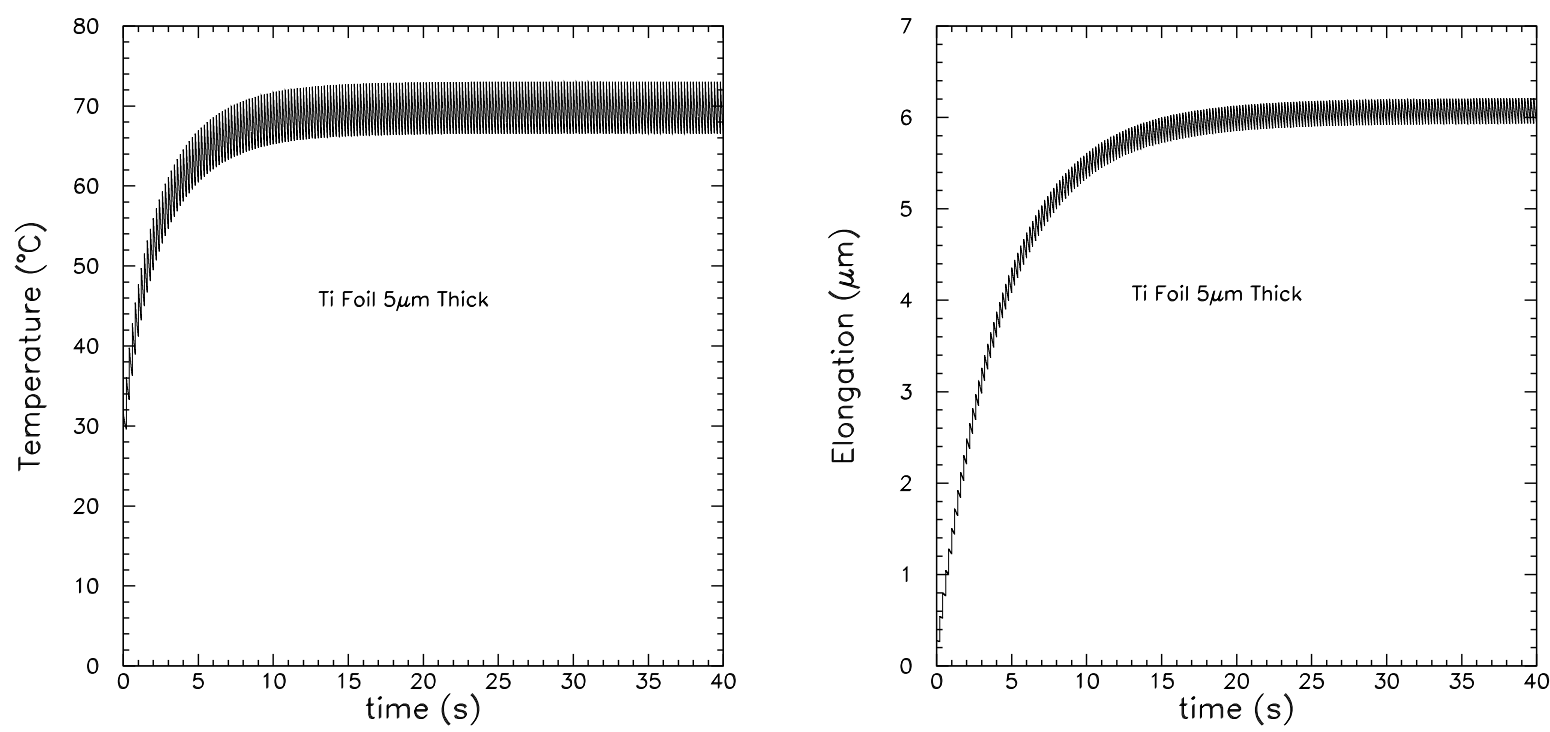

Figure 15: (left) Expected temperature rise at the center of the hottest foil during the upcoming test of the NuMI SEM in the MiniBoone beamline. (right) Net elongaton of the hottest Titanium foil during the MiniBoone test. 


\begin{tabular}{|c|c|c|c|c|c|c|}
\hline & \multicolumn{6}{|c|}{$400 \mathrm{MeV}$ linac } \\
\hline & $\begin{array}{c}\text { Thickness/ } \\
\text { Diameter }[\mu m]\end{array}$ & $-\frac{d E}{d x}$ & $\frac{\mathrm{MeV}}{\mathrm{gcm}-2}$ & $T_{\max }\left[{ }^{\circ} \mathrm{C}\right]$ & $\Delta T\left[{ }^{\circ} K\right]$ & $\begin{array}{c}\text { Fractional } \\
\text { elongation }\left[\times 10^{-4}\right]\end{array}$ \\
\hline Ti Foil & 5 & & 1.97 & 92.5 & 1.92 & 1 \\
\hline Ti Wire & 50 & & 2.06 & 191 & 2 & 3.9 \\
\hline W Wire & 25 & & 1.55 & 226 & 5.89 & 4.1 \\
\hline W Wire & 50 & & 1.57 & 277 & 5.97 & 5.4 \\
\hline W Wire & 75 & & 1.59 & 308 & 6.04 & 6.3 \\
\hline
\end{tabular}

\begin{tabular}{|c|c|c|c|c|c|c|}
\hline & \multicolumn{6}{|c|}{ MiniBoone } \\
\hline & $\begin{array}{c}\text { Thickness/ } \\
\text { Diameter }[\mu m]\end{array}$ & $-\frac{d E}{d x}$ & $\frac{\mathrm{MeV}}{\mathrm{gcm}-2}$ & $T_{\max }\left[{ }^{\circ} \mathrm{C}\right]$ & $\Delta T\left[^{\circ} K\right]$ & $\begin{array}{c}\text { Fractional } \\
\text { elongation }\left[\times 10^{-4}\right]\end{array}$ \\
\hline Ti Foil & 5 & & 1.07 & 73.1 & 6.5 & 0.52 \\
\hline Ti Wire & 50 & & 1.16 & 137 & 7.1 & 2.2 \\
\hline W Wire & 25 & & 0.9 & 157 & 21.4 & 2.3 \\
\hline W Wire & 50 & & 0.93 & 189 & 22.1 & 3.2 \\
\hline W Wire & 75 & & 0.94 & 204 & 22.3 & 3.6 \\
\hline
\end{tabular}

\begin{tabular}{|c|c|c|c|c|c|}
\hline & $\begin{array}{c}\text { Thickness/ } \\
\text { Diameter }[\mu m]\end{array}$ & $-\frac{d E}{d x}\left[\frac{M e V}{g c m^{-}}\right]$ & $T_{m a x}\left[{ }^{\circ} C\right]$ & $\Delta T\left[{ }^{\circ} K\right]$ & $\begin{array}{c}\text { Fractional } \\
\text { elongation }\left[\times 10^{-4}\right]\end{array}$ \\
\hline Ti Foil & 5 & 1.14 & 290 & 222 & 1 \\
Ti Wire & 50 & 1.23 & 400 & 240 & 3.4 \\
W Wire & 25 & 0.99 & 920 & 753 & 4.1 \\
W Wire & 50 & 1.02 & 990 & 775 & 5.4 \\
W Wire & 75 & 1.04 & 1035 & 791 & 6.3 \\
\hline
\end{tabular}

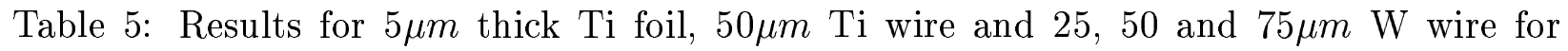
different beam line parameters. 

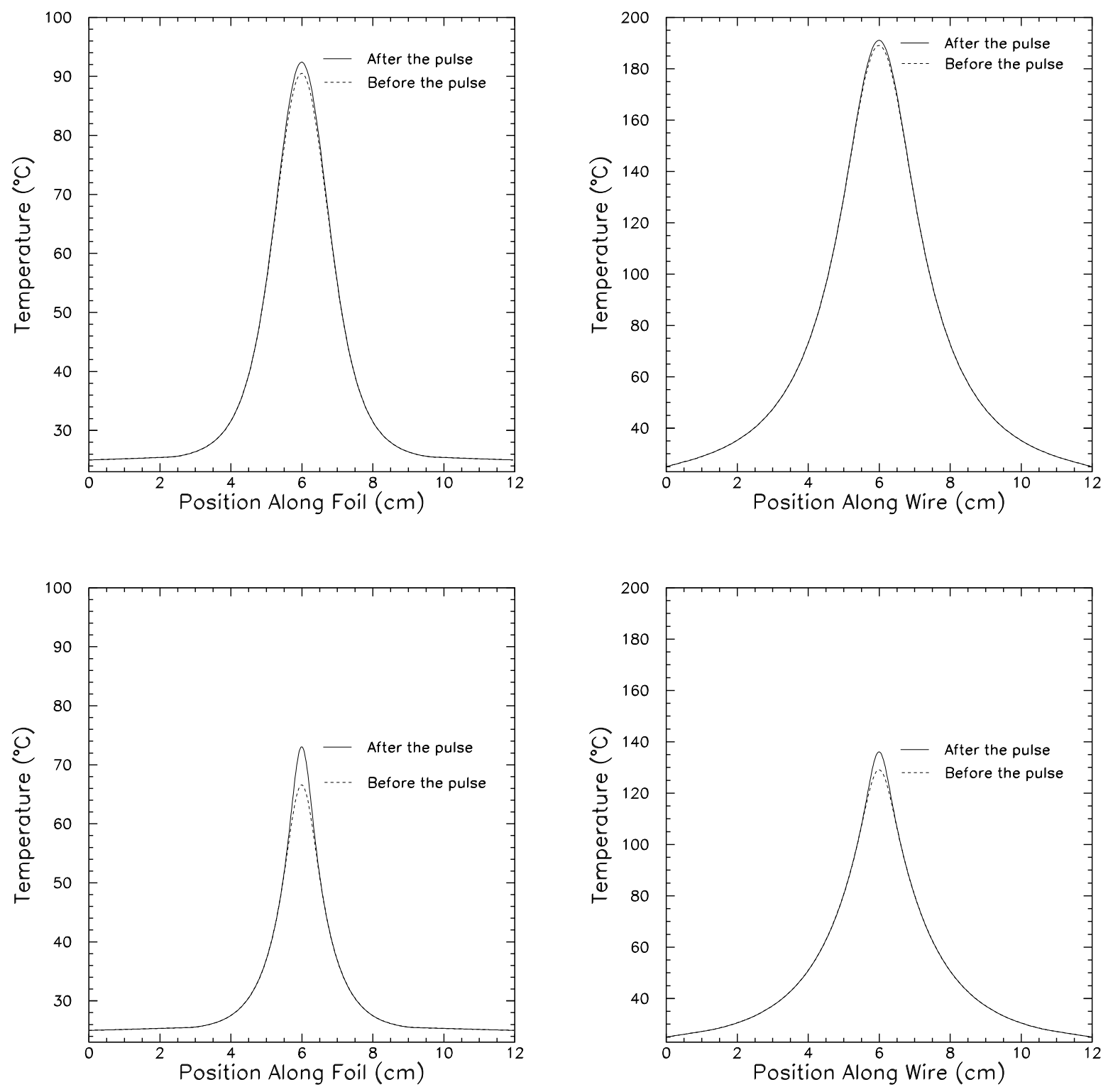

Figure 16: Temperature distribution along the foil/wire after reaching "equilibrium" calculated for Linac (top) and MiniBoone (bottom) beam line parameters. 


\section{Conclusion}

We have performed simulations of the beam heating in vacuum of various candidate SEM materials by the NuMI beam. Our estimates indicate that a Titanium foil SEM experiences acceptably small tension loss, provided that accordion springs in the material are employed to take up the thermal expansion. To keep the tension loss at the few percent level, the accordion springs should be able to accomodate up to 5-7 $\mathrm{mm}$ of expansion. Our estimates also suggest that a wire SEM without springs will not operate without tension loss, with the possible exception of a Carbon wire SEM.

\section{References}

[1] H.Bruining, "Physics and Applications of Secondary Electron Emission," (London, Pergammon Press), 1954.

[2] G. Ferioli \& R. Jung, "Evolution of Secondary Electron Emission Efficiencies of Various Materials Measured in the CERN SPS Secondary Beam Lines," CERN-SL-97-71(B1), talk presented at DIPAC '97.

[3] Handbook of Chemistry and Physics,68th Ed. Ed: Robert C. Weast. CRC Press, 19871988.

[4] http://www.kasap.usask.ca/server/kasap/Tables/Mechanical.html

[5] Handbook of Physical Quantities. Ed: Igor S. Grigoriev and Evgenii Z.Meilikhov. CRC Press, 1997.

[6] K. Hagiwara et al., "Review of Particle Properties," Phys. Rev. D66, 010001 (2002).

[7] B. Rossi, High Energy Particles, (Englewood Cliffs: Prentice Hall, Inc.) 1952. See especially Section 2.5, equations 2.5.4 and 2.5.6.

[8] National Institutes of Standards and Technologies, "Stopping Powers of Electrons, Protons, and Alpha Particles," physics.nist.gov/PhysRefData/.

[9] R.Valdiviez et al., Proceedings of 2001 Particle Accelerator Conference, p.1324.

[10] J.Krider \& C. Hojvat, Nucl. Instr. Meth. A247, 304 (1986).

[11] R. J. Roark, W. C. Young, "Formulas for Stress and Strain"

[12] Sacha E. Kopp, Marek Proga "Design of The Prototype NuMI Profile Monitor SEM", NuMI note NuMI-B-933 (2003).

[13] M.Plum, "Interceptive Beam Diagnostics: Signal Creation and Materials Interactions," Proc. $11^{\text {th }}$ International Beam Instrumentation Workshop (BIW04), AIP Conf. Proc. 732, pg. 23-46, Knoxville, TN (2004). 

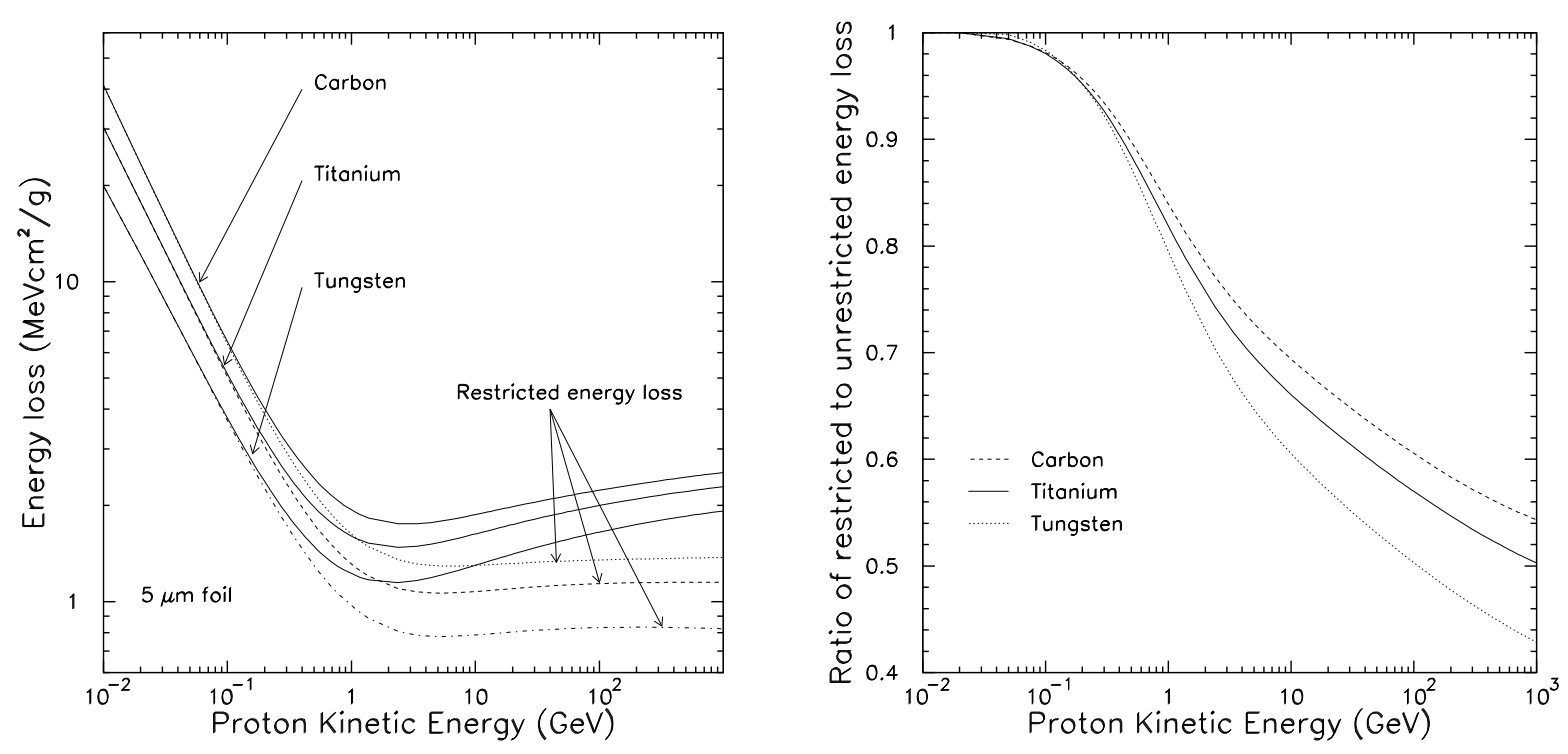

Figure 17: Restricted energy loss compared to unrestricted for $5 \mu \mathrm{m}$ foil.

\section{A Energy Loss}

Moderately relativistic charged particles other than electrons lose energy in matter primarily by ionization and atomic excitation. The mean rate of energy loss (or stopping power) is given by the Bethe-Bloch equation,

$$
-\frac{d E}{d x}=K z^{2} \frac{Z}{A} \frac{1}{\beta^{2}}\left[\frac{1}{2} \ln \frac{2 m_{e} c^{2} \beta^{2} \gamma^{2} T_{\max }}{I^{2}}-\beta^{2}-\frac{\delta}{2}\right]
$$

Here $T_{\max }$ is the maximum kinetic energy which can be imparted to a free electron in a single collision.

Integrating Equation 17 we can find the total 'continuous slowing-down approximation' range $\mathrm{R}$. This is for a particles which loses energy only through ionization and atomic excitation.

For a particle with mass $M$ and momentum $M \beta \gamma c, T_{\text {max }}$ is given by:

$$
T_{\text {max }}=\frac{2 m_{e} c^{2} \beta^{2} \gamma^{2}}{1+2 \gamma m_{e} / M+\left(m_{e} / M\right)^{2}}
$$

For density effect correction we use Sternheimer's parameterization:

$$
\delta= \begin{cases}2(\ln 10) x-\bar{C}) & x \geq x_{1} \\ 2(\ln 10) x-\bar{C}+a\left(x_{1}-x\right)^{k} & x_{0} \leq x<x_{1} \\ 0 & x<x_{0}(\text { nonconductor } s) \\ \delta_{0} 10^{2\left(x-x_{0}\right)} & x<x_{0}(\text { conductors })\end{cases}
$$



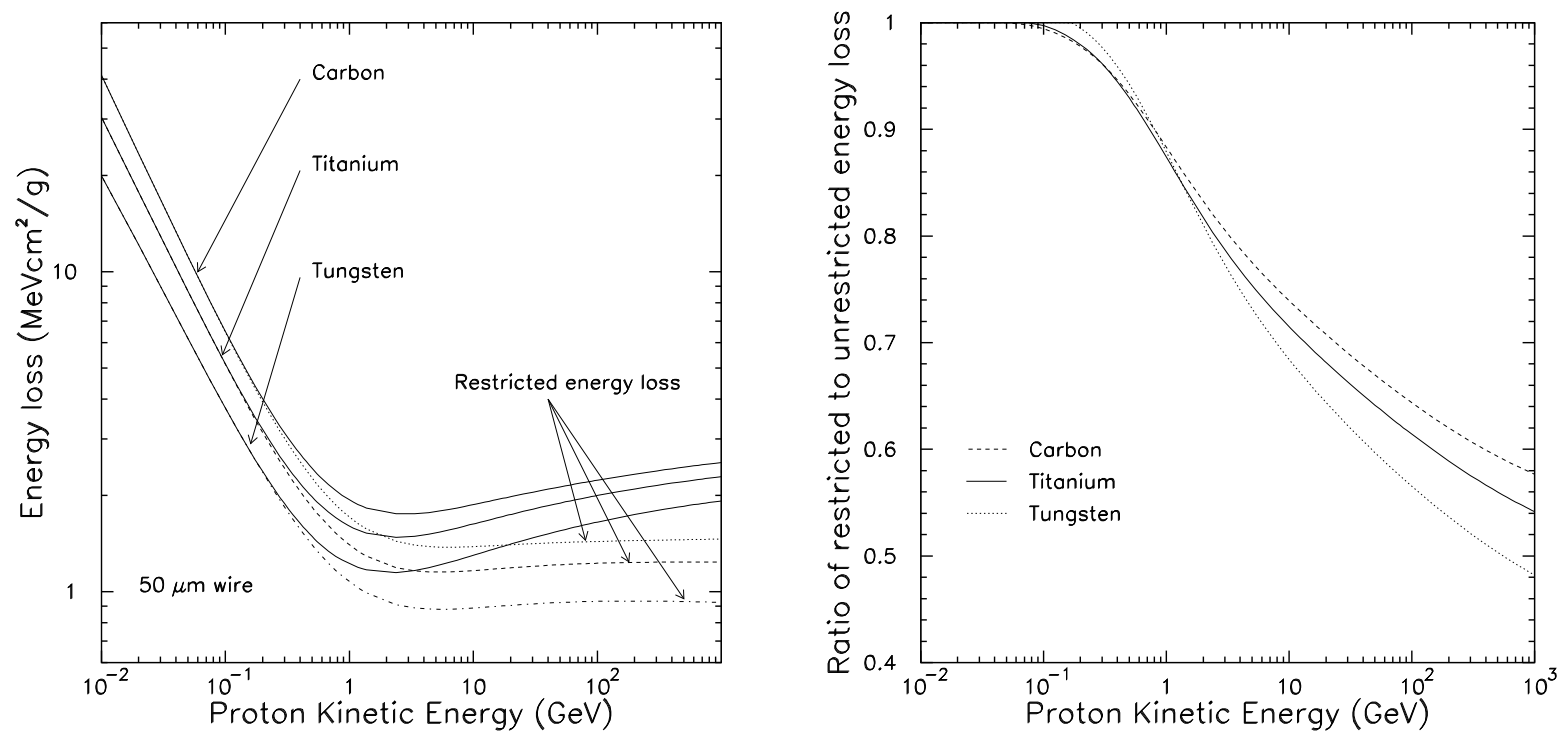

Figure 18: Restricted energy loss compared to unrestricted for $50 \mu m$ wire.

Here $x=\log _{10}(p / M c)$. Parameters for various materials considered as SEM candidates are listed in table 6 .

Proton passing through the material will deposit energy by ionizing particles. Since we are considering thin foil or wire as a target, only electrons with energy lower than some $T_{c u t}$ will stay inside the SEM material. Because of that we need to modify Equation 17. The restricted energy loss rate is:

$$
-\left(\frac{d E}{d x}\right)_{T<T_{\text {cut }}}=K z^{2} \frac{Z}{A} \frac{1}{\beta^{2}}\left[\frac{1}{2} \ln \frac{2 m_{e} c^{2} \beta^{2} \gamma^{2} T_{c u t}}{I^{2}}-\frac{\beta^{2}}{2}\left(1+\frac{T_{c u t}}{T_{\max }}\right)-\frac{\delta}{2}\right]
$$

Figure 17 and Figure 18 show the restricted and unrestricted energy loss and their ratio. Subtracting Equation 1 from Equation 17 we get the drop in energy deposition due to

\begin{tabular}{|c|c|c|c|c|c|c|}
\hline Material & $a$ & $k$ & $x_{0}$ & $x_{1}$ & $\bar{C}$ & $\delta_{0}$ \\
\hline Beryllium & 0.80392 & 2.4339 & 0.0592 & 1.6922 & 2.7847 & 0.14 \\
Carbon & 0.26142 & 2.8697 & -0.0178 & 2.3417 & 2.868 & 0.12 \\
Aluminum & 0.08024 & 3.6345 & 0.1708 & 3.0127 & 4.2495 & 0.12 \\
Titanium & 0.15662 & 3.0302 & 0.0957 & 3.0386 & 4.445 & 0.12 \\
Nickel & 0.16496 & 2.843 & -0.0566 & 3.1851 & 4.3115 & 0.1 \\
Silver & 0.24585 & 2.6899 & 0.0657 & 3.1074 & 5.063 & 0.14 \\
Tungsten & 0.15509 & 2.8447 & 0.2167 & 3.496 & 5.4059 & 0.14 \\
Gold & 0.09756 & 3.1101 & 0.2021 & 3.6979 & 5.5747 & 0.14 \\
\hline
\end{tabular}

Table 6: Parameters needed for density corrections. 
restricted energy loss:

$$
\Delta=K z^{2} \frac{Z}{A} \frac{1}{\beta^{2}}\left[\frac{1}{2} \ln \frac{T_{\text {max }}}{T_{c u t}}-\frac{\beta^{2}}{2}\left(1-\frac{T_{c u t}}{T_{\text {max }}}\right)\right]
$$

